\title{
The Multi-Level Model for quarks and leptons as the symbio- sis of Segal's Chronometry with the Standard Model
}

\author{
Alexander Levichev ${ }^{1,2, *}$ and Andrey Palyanov ${ }^{2}$ \\ 1 Sobolev Institute of Mathematics, Novosibirsk, Russia; alevichev@gmail.com \\ 2 Ershov Institute of Informatics Systems, Novosibirsk, Russia; palyanov@iis.nsk.su \\ * Correspondence: alevichev@gmail.com
}

\begin{abstract}
The Multi-Level Model (=MLM) was suggested by A. Levichev as a description of quarks and gluons. The review recalls MLM-terminology while MLM-findings (for quarks and leptons) are compared to the experimental data as accepted by the Standard Model (=SM). MLM is based on Segal's compact space-time $U(2)$ and on the sequence of embeddings: $U(2)$ into $U(3), U(2)$ into $U(4)$, etc. These groups were called the levels (of matter): $U(2)$ - the $0^{\text {th }}$ (that is, our mundane world), $U(3)$ - the $1^{\text {st }}, \mathrm{U}(4)$ - the $2^{\text {nd }}$, etc. Such a convention matches the SM-quarks' generations list. Each SM-quark is viewed either as a sunken proton, or as a captured proton. The MLM-proton is elementary and indestructible (hence no need for confinement). For MLM-quarks, in terms of the notion of a ruling group, flavor and color are defined mathematically. The number of colors (and of flavors) is level-dependent. For levels $U(3)$ through $U(6)$ the correspondence with the SM-quarks is established. Three new quarks and two new leptons are predicted. The SM-puzzle of quark and lepton generations is solved. Using the Han-Nambu scheme, the notion of the quark's electric charge is expressed in terms of color charges. The original part of the review suggests studying the proton's properties (like mass, shape and inner pressure) on the basis of its wave function.
\end{abstract}

Keywords: Standard and Multi-Level Models; Wigner-Segal method; quark matter; generations of quarks and leptons; chronometric proton's wave function; proton shape

\section{Introduction}

In [1], with a goal of understanding the quark-gluon media, the Multi-Level Model (MLM, for short) was suggested. Below, starting with the next section, MLM's main tenets and terminology are recalled (mostly from [1, 2]). To mathematically model elementary particles, the Chronometric Theory is applied throughout (see, especially, the section Indecomposable Elementary Particle Associations in [3]). The method of [3] can be interpreted as the one generalizing the suggestion by Wigner [4] to model particles on the basis of certain Poincare group (denote this group by $P$ ) representations. In [3] Segal treats certain representations of $G=S U(2,2)$. This latter group (sometimes called the conformal group) contains both the (11-dimensional) extended Poincare group $P^{+}$and its (10-dimensional) subgroup $P$. In [5] it was suggested to name the approach of [3] as the Wigner-Segal method. The abbreviation SM stands for the (currently widely accepted) Standard Model. In [1, 2] the MLM was described as an alternative to the SM. On the basis of further studies (see [5-10]), MLM can now be viewed as the symbiosis of Segal's Chronometry with SM. The main purpose of the current review is to put together concise and, hopefully, convincing arguments in order to support such a far-reaching view.

\section{From Chronometry to the MLM-quarks of the $U(3)$-level}

First, the Segal's Chronometry has to be outlined. Recall that A. Levichev has surveyed it in [11] (in Russian). Also, it is surveyed in [12] which is online.

Mathematically, Chronometry deals with a slightly larger totality of space-time events than the Minkowski space-time $M$ has. Namely, the compact chronometric world $\mathbf{D}$, 
as a manifold, is the unitary group $U(2)$ which is defined by formula (3.3) of [9]. (This suffices for our purposes. To eliminate closed time-like loops one has to move to the universal covering group.) Here (and on) we use world as a synonym of space-time. The imbedding of $M$ into $\mathbf{D}$ via the Caley transform is well-known, see formula (5.2) of [12]. The Lorentzian metric (or inner product) in $\mathbf{D}$ was introduced by Segal (and is given in Section 3.1 of [12]). This metric is left-invariant as well as right-invariant on the Lie group $U(2)$. The above mentioned Poincare group $P$ is the totality of all isometries of the (pseudoEuclidian) $M$. The group $P^{+}$can be obtained from $P$ by adding scaling transformations; hence $P$ is a subgroup of $P^{+}$.

The main group of (causal) transformations in $\mathbf{D}$ is (15-dimensional) $S U(2,2)$, see formula (2.2) from [2]. Certain representations (see [3]) of this group give rise to chronometric particles. The adjective chronometric indicates that we are within the Chronometry. The particles suggested by this theory should be compared to relativistic particles, where the (10-dimensional) Poincare group $P$ is the main symmetry group, or to Galilean particles, when (also 10-dimensional) Galilean group is the main one. For our research it is important to remember that the SM deals with relativistic particles while the MLM, since it uses many findings of Segal's School, treats chronometric particles, essentially. In [2] (Section 2) it was outlined how a property (one or another) of a chronometric particle can be interpreted in relativistic terms.

For more details on Chronometry a reader is advised to visit Section 2 of [2] or suitable references therein. However, on the basis of the findings in [10], we point out that certain corrections (of what Segal claimed in [3], and what was reproduced from [3] in Section 2 of [2]) have to be made. The research [10] can be viewed as a discussion of, and supplement to, Segal's list of chronometric elementary particles of spin 1/2 [3]. The last article is in some sense a summary of Segal's findings, and it is just 5 pages long. In [3], there are few, if any, clues of how to obtain results outlined in it. One of [10]'s goals was to prove (some of) Segal's statements. The most remarkable of these is that there are four elementary chronometric particles of spin $1 / 2$. Namely, there is a massive neutral particle named the exon, the electron $e$, and two types of neutrino (interpreted as $v_{e}$ and $v_{\mu}$ ). We failed to prove that (but see what we say below, on this page). In Segal's theory, a particle (e.g. each of the above) is mathematically associated with an irreducible unitary positiveenergy representations of the symmetry group $G$ (in our case, the conformal group SU(2; $2)$ ). Let us stress that when we, below, associate certain mathematical objects with specific particles; we try (as much as possible) to stay in line with what Segal has done before in this regard. Here is the only significant exception to the above: around 2010 A. Levichev suggested [11] (Section 7) that it is rather the proton $p$ than a hypothetical neutral particle, the exon.

Later that suggestion gave birth to Levichev's Multi-Level Model of quarks, MLM [1, 2] which claims that each quark can be viewed as if it is a state of the proton. Segal himself thought [3] (p. 994) that the exon could be the main ingredient of both the neutron and the proton. The research [10] displays an algebraic model (in terms of the composition series, which is inescapable when one deals with an indecomposable representation), in which there is just one, rather than two, as in the Segal article, light (i.e. massless) particle.

It is worth mentioning that an earlier conjecture by Segal (about the number of chronometric spin 1/2 particles) was in compliance with the findings in [10]: see [13] (Th. 16.7.10) which worked with a 3-step composition series. The representation is a limiting case of representations studied by Jakobsen in [14], where, purely algebraically, a 3-step series may be obtained after-the-fact. Later, Segal's original (that is, the one of [13]) conclusion has been withdrawn: [3] (Table 1) states (without proof) the existence of the 4-step composition series. Overall, the authors of [10] follow the approach of [3] (see the Section Indecomposable Elementary Particle Associations ibid). Below (in this Section), we will give more details from [10]. In particular, in the general context of how to mathematically define the notion of an elementary particle, we will comment on the transition from the renowned Wigner method to (what have been coined in [5] as) the Wigner-Segal method. Meanwhile we only state the following (it is an extract from [10]): there is a certain 
(infinite-dimensional) Hilbert space $F_{p}$ (of functions on the Minkowski space-time $M$ ) which is interpreted as the set of all (theoretically possible) states (or wave functions) of the chronometric proton $p$ (see formula (20) of [10] and Theorem 3.1 of [9]). The group $G=$ $S U(2,2)$ acts on $F_{p}$. According to the terminology of [9] (Remark 4.1), this $G$ is the ruling group, $\mathrm{Gr}$, since it rules (or governs) the behavior of the particle (the latter thus being the proton $p$ in our case).

We are now in a position to start a description of the MLM. This model plays with the sequence of canonical (that is, based on principal minors of the matrices involved) embeddings of groups: $U(2)$ into $U(3), U(2)$ into $U(4), U(2)$ into $U(5)$, etc. These groups were called levels: $U(2)$ is the $0^{\text {th }}$ level, $U(3)$ - the $1^{\text {st }}, U(4)$ - the $2^{\text {nd }}$, etc. In our Section 3 we will see that such a convention matches the standard quarks' generations list. Recall that each matrix group $U(\mathrm{n})$ is defined quite similarly to how $U(2)$ was defined by formula (3.3) of [9]. Embeddings $\mathrm{A}_{12}, \mathrm{~A}_{13}, \mathrm{~A}_{23}$ of (the Segal's compact cosmos) $\mathbf{D}=U(2)$ into $U(3)$ were introduced as follows: under each of these three embeddings a matrix $Z$ from $D$ becomes a certain principal 2 by 2 minor of the corresponding 3 by 3 matrix from $U(3)$. Namely, let us denote by $\mathrm{D}_{12}$ the image of the embedding $\mathrm{A}_{12}$ where $\mathrm{A}_{12}$, itself, is defined as follows (also, these embeddings are illustrated in the top portion of Figure A1):

Each $Z$ from $D$ is now an upper 2 by 2 principal minor of the 3 by 3 matrix $A_{12}(Z)$ in $U(3)$; the third diagonal entry of $\mathrm{A}_{12}(Z)$ is 1 ; in the $\mathrm{A}_{12}(Z)$, all other entries vanish. The two remaining embeddings, $\mathrm{A}_{13}$ and $\mathrm{A}_{23}$ are defined quite similarly. Clearly, $\mathrm{D}_{12}, \mathrm{D}_{13}$, and $\mathrm{D}_{23}$ are $U(2)$-subgroups in $U(3)$. Recall that the group $U(2)$ is closed w.r.t. the complex conjugation, and w.r.t. the matrix transposition. The transposed matrix $Z^{\mathrm{T}}$ can be viewed as a mirror of $Z$ w.r.t. reflection in the principal diagonal. From this it follows that each of the $\mathrm{D}_{12}, \mathrm{D}_{13}, \mathrm{D}_{23}$ is invariant w.r.t. any of the two mentioned operations in $U(3)$. Also, to enumerate all $D_{i j}$, it is enough to consider the cases $\mathrm{i}<\mathrm{j}$, only. We supply each $\mathrm{D}_{\mathrm{ij}}$ with a Lorentzian metric by the demand that each $\mathrm{A}_{\mathrm{ij}}$ be an isometry.

In the totality of all $m$ by $m$ matrices, introduce $P_{m}$, as the symmetry w.r.t. the opposite diagonal. Clearly, when $Z$ is in $U(2)$, then $\mathrm{P}_{2}(Z)$ is also in $U(2)$. From this it follows that the subgroup $\mathrm{D}_{13}$ is $\mathrm{P}_{3}$-invariant in $U(3)$ while $\mathrm{P}_{3}\left(\mathrm{D}_{12}\right)=\mathrm{D}_{23}, \mathrm{P}_{3}\left(\mathrm{D}_{23}\right)=\mathrm{D}_{12}$. That enables us to view the embeddings $\mathrm{A}_{12}$ and $\mathrm{A}_{23}$ as equivalent (one becomes the other when composed with $\mathrm{P}_{3}$ and $\mathrm{P}_{2}-$ see Figure A1). This relates to the 'presence of two SM- $u$-quarks in' a proton, while the $A_{13}$ relates to the presence of an SM- $d$-quark in that proton. These embeddings make it possible to introduce a notion of a flavor of a MLM-quarks of level $U(3)$. The last two phrases are 'non-mathematical', we relate to physics here. We discuss this in more detail later in this section (right after Theorem 1).

Definition 1. Below, in many instances, we use the name cell for each of these $\mathrm{D}_{\mathrm{ij}}$.

This seems to be a reasonable way of attaching names: compare to such a straightforward (but the one which is less 'compact') alternative as of "a minor (one or another) of the (corresponding) matrix of a certain size". It should always be stated (or should be clear from the context) which $U(\mathrm{n})$-level such a cell is considered to be in.

In [2] (Section 3), similarly to the way of introducing (in $U(3)$ ) the $U(2)$-subgroups $D_{12}, D_{13}, D_{23}$; the $S U(2,2)$-subgroups $G_{12}, G_{13}$, and $G_{23}$ of $S U(3,3)$ have been defined.

Definition 2. Below, in many instances, we use the name ruling group, or the $r$ group, for each of these $G_{i j}$ (the latter being an $S U(2,2)$-subgroup of $S U(n, n)$, in general). It should always be stated (or should be clear from the context) which $U(\mathrm{n})$-level such an $r$ group is associated with. From [5], we now reproduce the following statement [5] (Theorem 4.1).

Theorem 1. An action of each of the subgroups $\mathrm{G}_{12}, \mathrm{G}_{13}$, and $\mathrm{G}_{23}$ on any of the subgroups $\mathrm{D}_{12}, \mathrm{D}_{13}$ and $\mathrm{D}_{23}$ is defined. In particular, each of the following three actions, $\mathrm{G}_{12}$ on $\mathrm{D}_{12}, \mathrm{G}_{13}$ on $\mathrm{D}_{13}$, and $\mathrm{G}_{23}$ on $\mathrm{D}_{23}$, is the linear-fractional one.

Let us now say more about the mathematical meaning (and about the physical interpretation) of the following phrase (from above): "The embeddings $\mathrm{A}_{12}$ and $\mathrm{A}_{23}$ relate to the presence of two SM- $u$-quarks in a proton, while the $\mathrm{A}_{13}$ relates to the presence of an SM-d-quark in that proton". 
First, let us recall that (according to the SM) a proton consists of two $u$-quarks and of one $d$-quark. The detection of three point-like centers (of highly inelastic electron-proton scattering, see [15]) served as an experimental basis for such a conclusion about the structure of a proton. However, after several decades of intense search, the majority of the Physics community has submitted to the view that "free quarks cannot be detected."

Before we introduce the MLM-quarks in $U(3)$ (and later, with the increase of $n$, in each of the levels $U(n)$ ), we need to present more mathematical findings (from [9, 10], mostly) related to Chronometry. Above, closer to the beginning of the Section, we have been discussing chronometric particles of spin $1 / 2$. They originate from an induced representation of the group $G=S U(2,2)$ defined by formula (5) from Section 2 of [10]. In [10] (Section 9), it was concluded that exactly three chronometric spin $1 / 2$ fermions have been mathematically detected (and interpreted as the proton $p$, electronic neutrino $v_{\mathrm{e}}$, and electron $e$ ). It was mentioned in [10] (right before Remark 1.1 there) that such a conclusion did not involve the MLM, per se. On the basis of formula (16) from [10] (Section 4), the space $F$ of the induced representation (sometimes referred to as the spannor representation) of the group $G=S U(2,2)$ has been introduced. In $F$, there exist two non-trivial invariant subspaces with no invariant complement. One of those subspaces was denoted by $F_{p}$ and it was supplied with a Hilbert space structure. On the basis of the findings in [14, 16], the following statement has been proven (see Section 5 of [10]):

Theorem 2. The restriction of the induced representation to $\mathrm{F}_{\mathrm{p}}$ is unitary and irreducible. In $F_{p}$, the energy-positivity condition holds.

The space $F_{p}$ has been interpreted as the totality of all (theoretically possible) wave functions of the chronometric proton. Notice that $F_{p}$ is of conformal weight (or conformal dimension) 5/2, see [10] (Section 5).

Remark 1. Let us repeat that in [10] (Section 6), it was stated that $F_{p}$ does not have an invariant complement. It means that we deal with the case where the Wigner method is not applicable. According to the Wigner-Segal method, we now have to deal with the quotient space $W=F / F_{p}$ and with the factor-representation in it. In [10] (Section 6), a minimal non-trivial invariant subspace $F_{v}$ in $W$ has been introduced, $F_{v}$ has been supplied with the unitary structure and it has been interpreted as the totality of all wave functions of the chronometric electronic neutrino. In [10] (Section 7), the quotient space $W / F_{v}=F_{e}$ has been interpreted as the totality of all wave functions of the chronometric electron (since the corresponding factor-representation turned out to be irreducible and unitarizable, and the conformal weight is $3 / 2$ now ). This was the final step in the proof of the main finding of [10]: there are exactly three elementary chronometric spin $1 / 2$ particles.

Remark 2. Having in mind Remark 9.1 of [10], from now and on it seems plausible to associate the Hilbert space $F_{v}$ with the electronic antineutrino, rather than with electronic neutrino. Let us recall that the authors of [10] followed Segal (see [3]) and they interpreted this ('middle') sector of the 3-step composition series as the one corresponding to the electronic neutrino but (also in [10]) they have envisaged a possibility of the antineutrino interpretation. Such an interpretation can serve as a mathematical reason allowing a return (as it has been claimed in [17]) to an 'old model' for the neutron as consisting of a proton, of an electron, and of an electronic anti-neutrino - see the $U(2)$-part of the Figure A4.

For the level $U(3)$, recall that each $\mathrm{A}_{\mathrm{ij}}$ is an isometry, and that each $\mathrm{D}_{\mathrm{ij}}$ is a space-time isometric to the Segal's compact cosmos D. From here (and on the basis of both the above Theorem 2 and of its interpretation), we conclude that a spin $1 / 2$ fermion ('living in' $\mathrm{D}_{\mathrm{ij}}$ ) is mathematically defined. If $\mathrm{D}_{12}$ or $\mathrm{D}_{23}$ is a support of its wave functions then, as part of the MLM, we associate this fermion with an $u$-quark. If $\mathrm{D}_{13}$ is such a support - then the particle is announced to be a $d$-quark. It means that in the MLM we have introduced two flavors for quarks of the $1^{\text {st }}$ level, $U(3)$, and (by merely keeping the SM-terminology) we have established the correspondence of MLM-quarks to the SM-quarks, and vice versa. It is assumed that a Hilbert structure in the corresponding spaces $H_{\mathrm{ij}}$ is introduced via the isometries $A_{i j}$ from the original $H=F_{p}$. For each of our fermions of the level $U(3)$, its $r$ group $G$ can be any of $G_{12}, G_{13}$, or $G_{23}$. Such a convention was a mathematical basis for 
defining the notion of a color of an MLM-quark (see Sections 3 and 4 of [5], as well as our Section 5, below ). Clearly, each MLM-quark is as fully described, as the chronometric proton was - see our Theorem 2, from above. One is thus tempted to think of an MLMquark "as if" being a state of the (chronometric) proton.

Remark 3. Our "as if" cannot be dropped here; at least (if dropped), additional explanation should be provided. Here is why: a proton is described by one pair $(H, G)$ while an MLM-quark - by another. In this regard, let us recall that the SM allows usage of different (however, of isometric, only) Hilbert spaces of wave functions for the same particle. Hence, let us recall that the following holds: the two $r$-groups (one for the proton, another for the MLM-quark) consist of matrices of different sizes; in the first case - those are 4 by 4 matrices (since our proton lives in $U(2)=\mathrm{D}$ ), while the $r$-group $G$ (for the quark) is a subgroup of $S U(3,3)$. The latter means that the corresponding matrices are 6 by 6 ones. We have thus explained why, formally, we should not view MLM-quarks as states of a proton. For a pair of different MLM-quarks (of the same MLM-level), the respective $r$-groups (consisting of the matrices of the same size), are, however, different subgroups of $S U(3,3)-$ or of $\operatorname{SU}(\mathrm{n}, \mathrm{n})$, if $U(\mathrm{n})$ is the level in question. To finalize the current remark, we would like to stress that the main reason to not consider a quark as the state of a proton is that the two particles 'live' in different MLM-levels.

Rather than to use 'a state of a proton' terminology for an MLM-quark, let us word the process (of highly inelastic electron-proton scattering) in question as follows. As the result of such a scattering, proton gets (from $U(2)$ ) to a 'deeper' level, $U(3)$. In $U(3)$ it gets to one particular cell (of the total of three available ones: $\mathrm{D}_{12}, \mathrm{D}_{13}$, or $\mathrm{D}_{23}$ ) thus becoming an MLM-quark. Recall that the notion of a cell has been mathematically introduced above, in Definition 1. In terms of Physics, a possible description (see our Subsection 6.4, below) could use the following wording: "our proton pushes 'deeper' (that is, to the U(4)-level) the 'former occupant' of this cell. However, to better understand such a wording, it is recommended to read our Section 5, first.

Since we have introduced an equivalence (under operators $P_{3}$ and $P_{2}$ - see Figure A1) between $\mathrm{D}_{12}$ and $\mathrm{D}_{23}$, we only have two flavors rather than three. The word combinations, 'gets to one particular cell' and 'becomes an MLM-quark', merely mean that this stage of scattering has to be described in terms of the Multi-Level Model rather than using the SMlanguage. In a formal agreement with the SM, our description is that of elastic scattering (of the original electrons hitting the proton) on quarks (in our case - on MLM-quarks). In particular, within the $U(3)$ level, we should use another Hilbert space and another $r$ group. These last two issues might be less, or even not at all, significant in comparison to a change of state of our proton as it becomes an MLM-quark. Such a change seems to be an unavoidable one.

A reader is now advised to go to Figures B1, B2, and B3 in order to get a clue on what a state of a proton may look like. Seemingly, the thus suggested description of the highly inelastic electron-proton scattering does not, per se, contradict to detection of 'three pointlike components in a proton'. Also, in the MLM-approach one can apply the combinatorial SM-methods to calculate relations between certain scattering probabilities (see, for example, Section 6 of [2]).

Let us provide more MLM-details. If our proton gets into the cell $\mathrm{D}_{12}$, then (instead of the former $F_{p}$ ) we have to exploit the space $F_{12}$ of wave functions defined on $D_{12}$, rather than on the original D.

Let us recall that due to an isometry $\mathrm{A}_{12}$ between $\mathrm{D}$ and $\mathrm{D}_{12}$, the Hilbert spaces $F_{p}$ and $F_{12}$ are unitarily equivalent. In $[5$, Section 3$]$ the notation $q(1 ; 1,2)$ has been used for such an MLM-quark: the first ' 1 ' is the level number (here it means the $U(3)$ level), while the pair $(1,2)$ specifies the cell (being D 12 here).

\section{The MLM-quarks of levels $U(4)$ and $U(5)$}

Introduce embeddings of $\mathrm{D}=U(2)$ into $U(4)$, first. Here they are (see Figure A2 of Appendix $A)$ : $A_{12}, A_{13}, A_{14}, A_{23}, A_{24}, A_{34}$; the notation mimics the one which has been already 
used in the (3)-case. To determine equivalences, consider the (applicable to any 4 by 4 matrix) operator $\mathrm{P}_{4}$ : the symmetry w.r.t. the opposite diagonal. Clearly (as the Figure A2 illustrates), $\mathrm{A}_{12}$ is equivalent to $\mathrm{A}_{34}$, and $\mathrm{A}_{13}$ is equivalent to $\mathrm{A}_{24}$. Each of the subgroups $\mathrm{D}_{14}$ and $\mathrm{D}_{23}$ is $\mathrm{P}_{4}$-invariant. Relate $\mathrm{A}_{14}$ to an SM-quark s, and $\mathrm{A}_{23}$ - to an SM-quark $c$. At this (that is, at the second) level, $\mathrm{A}_{12}$ (which is equivalent to $\mathrm{A}_{34}$ ) relates to an SM-quark $u$ while $\mathrm{A}_{13}$ (equivalent to $\mathrm{A}_{24}$ ) relates to an SM-quark $d$. Hence, SM-quarks of both generations (one and two) 'live' on the $2^{\text {nd }}$ MLM-level, $U(4)$.

Now, for an interested reader (even if this person does not have much knowledge about currently known elementary particles), we give an overview of SM-quarks' generations, as well as of the topic in general. Namely, by 2018 it was known that there exist (at least) three generations of quarks and three generations of leptons. These fundamental particles are thought to be adequately modeled as the 'point-like' ones. Both quarks and leptons have spin $1 / 2$ which means that they are fermions. By convention, a fermion is a particle with half-integer spin, while a boson is a particle with an integer spin. Mathematically, a spin of a particle is a certain constant which is present in the (describing this very particle) representation of the group $G$ - see, i.e. [18](p. 348). By $G$ here (as well as above), we mean the main group of transformations acting in the space-time which our particle 'lives in'. The word generation is part of the SM-terminology. (As it should be already clear, generations, essentially, are naturally 'built into' the MLM.)

According to SM, the first generation of quarks consists of $u$ and $d$; the first generation of leptons consists of the electronic neutrino $v_{\mathrm{e}}$, and of the electron $e$. The second generation: the quarks $c$ and $s$; the leptons - muonic neutrino $v_{\mu}$ and the muon $\mu$. The third generation: the quarks $t$ and $b$; the leptons - tau neutrino $v_{\tau}$ and the tau particle $\tau$. Also, for each particle, there is an anti-particle. The SM-quarks are considered to have color and to have (fractional) electric charge. (Within the MLM, we will discuss both notions (color and electric charge) below, see our Section 5). The SM-leptons (we have just listed all of them) are colorless particles having charge zero - as regards neutrinos, and charge 1 (or negative 1) - as regards the remaining leptons. Matter is built of atoms which are believed to be built of the first generation fermions. Within the SM, it is not quite clear why Nature needs the $2^{\text {nd }}$ and the $3^{d}$ generation fermions. As it will be seen below, the MLM subdivides the above fermions into generations by its very structure. It is very difficult to create and to detect fermions of generations 2 and 3: powerful charged particles' accelerators are to be used for that. Besides, the created particles decay almost instantly. Within generations two and three, only neutrinos are believed to have the unlimited lifetime. Currently the search for the $4^{\text {th }}$ generation of particles is going on (our Figure A3 indicates 'where' to look for quarks of the $4^{\text {th }}$ generation).

Part of the above presented description of fermions and of their role as seen by the $\mathrm{SM}$ is a (translated from Russian) portion of http://phys.vspu.ac.ru/forstudents/TSOR/Kutseva/pokolenie leptonov i kvarkov.html.

We now return to the MLM-description of quarks. Here is the list of all $\mathbf{D}=U(2)$ embeddings into $U(5)$ : $A_{12}, A_{13}, A_{14}, A_{15}, A_{23}, A_{24}, A_{25}, A_{34}, A_{35}, A_{45}$. To get a quick grasp of the picture in question, our reader can easily think of an illustration, similar to Figure A2. Clearly, $\mathrm{P}_{5}\left(\mathrm{D}_{12}\right)=\mathrm{D}_{45}$; we relate it to an SM-u-quark. $\mathrm{P}_{5}\left(\mathrm{D}_{13}\right)=\mathrm{D}_{35}$, we relate it to an SM- $d$ quark. $P_{5}\left(D_{14}\right)=D_{25}$, we relate it to an SM-s-quark. $P_{5}\left(D_{23}\right)=D_{34}$, we relate it to an SM-cquark. Each of the following two subgroups, $\mathrm{D}_{15}$ ( $t$-quark) and $\mathrm{D}_{24}$ (b-quark), is $\mathrm{P}_{5}$-invariant. Hence, 'MLM-copies' of the SM-quarks of all three generations 'live' on the $3^{\mathrm{d}}$ MLMlevel $U(5)$.

From now and till the end of Section 4, let us write the word 'quark' everywhere instead of the earlier used 'MLM-quark'. Recall (from $[2,5])$ the notation $q(n ; i, j)$ for the corresponding quark of the $n$-th level; one can always assume that $\mathrm{i}<\mathrm{j}$. Such a convention results in the following relations between MLM- and SM-quarks:

$q(1 ; 1,2)=q(1 ; 2,3)=u, q(1 ; 1,3)=d ; q(2 ; 1,2)=q(2 ; 3,4)=u, q(2 ; 1,3)=q(2 ; 2,4)=d$,
$q(2 ; 2,3)=c, q(2 ; 1,4)=s ; q(3 ; 1,2)=q(3 ; 4,5)=u, q(3 ; 1,3)=q(3 ; 3,5)=d$,
$q(3 ; 2,3)=q(3 ; 3,4)=c, q(3 ; 1,4)=q(3 ; 2,5)=s, q(3 ; 2,4)=b, q(3 ; 1,5)=t$, "top quark". 
Recall that ' 2 ' (in, say, $q(2 ; 1,4)$ ) is the number of the level, while the pair $(1,4)$ indicates the cell D14. Also, compare to an example at the end of Section 2 and see Figure A3.

Remark 4. The SM-quarks of higher generations were detected later (than SM-quarks of lower generations), with the increase of the accelerators' typical energies. Hence, it is natural to interpret the 'deepening', with the increase of $n$, of the $U(\mathrm{n})$-levels as corresponding to the increase of the scattering typical energy.

As regards the total number of MLM-quarks at a given MLM-level, we reproduce (from $[1,2]$ the following

Theorem 3. On the level U(n), suppose an U(2)-subgroup $D_{i j}$ be not $P_{n}$-invariant. Then $\mathrm{D}_{\mathrm{ij}}$ corresponds to a quark from a lower level. The recurrent (1) and explicit (2) formulas (for the total number $\mathrm{m}_{n}$ of quarks at the U(n)-level) hold:

$$
\begin{aligned}
& \mathrm{m}_{2}=1, \mathrm{~m}_{\mathrm{n}}=\mathrm{m}_{\mathrm{n}-1}+[\mathrm{n} / 2], \\
& \mathrm{m}_{\mathrm{n}}=\{\mathrm{n}(\mathrm{n}-1) / 2+[\mathrm{n} / 2]\} / 2 .
\end{aligned}
$$

Here, $[x]$ denotes the greatest integer part (roof) of a real number $x$.

\section{On fractional electric charges of SM-quarks and the Han-Nambu scheme}

Most part of this Section's content is a translation of (certain) portions of [5].

According to the Standard Model, the electric charge of each quark $u$ (or $c$, or $t$ ) is $2 / 3$, while the electric charge of each quark $d$ (or $s$, or $b$ ) is minus $1 / 3$. There is an approach with integer quarks' charges, [19]. Such an approach is known as the Han-Nambu scheme (in [19] there is a reference to the original Han-Nambu publications). Our current section reproduces (from [5]) the adaptation of the Han-Nambu scheme to the MLM.

Consider the $U(3)$-level, first. Let us insert our notation into the following Table (which we reproduce from p.1 of [19]).

Table 1.

\begin{tabular}{cccc}
\hline $\mathbf{f} \backslash \mathbf{c}$ & $\mathbf{1 2}$ & $\mathbf{2 3}$ & $\mathbf{1 3}$ \\
\hline$u: 12,23$ & 1 & 1 & 0 \\
$d: 13$ & 0 & 0 & -1 \\
\hline
\end{tabular}

In the first row of this Table, $\mathbf{f}$ is for flavor, that is, $u$ or $d$; $\mathbf{c}$ is for color (not to be confused with the notation for the quark $c$ !). The last three entries in the first row are abbreviations for $G_{12}, G_{23}$, and $G_{13}$. This notation for corresponding $S U(2,2)$-subgroups of $\operatorname{SU}(3,3)$ was introduced in our Section 2; however, a 'shorter' one, 12, 23, 13 should not be confusing neither. Similarly (in the beginning of the Table's second row) we use 12, 23 instead of $\mathrm{D}_{12}, \mathrm{D}_{23}$, etc.

Before we state a possible 'color-related' interpretation of the above Table 1, let us provide more details (from [5], mostly) on how the subgroups $G_{\mathrm{ij}}$ have been introduced.

It is well-known that each matrix $g_{\mathrm{n}}$ (in $\left.G_{\mathrm{n}}=S U(\mathrm{n}, \mathrm{n})\right)$ is composed from $n$ by $n$ blocks $A_{\mathrm{n}}, B_{\mathrm{n}}, C_{\mathrm{n}}, D_{\mathrm{n}}$. Also, there is a fundamental (linear-fractional) $G$ n-action on $U(\mathrm{n})$; see more details about it in [20] (Section 2.1). For the $U(3)$-level, recall (from our Section 2) the embedding $A_{12}$. It designates the rows 1 and 2, and it designates the columns 1 and 2. Such a designation (choice) of rows and columns uniquely specifies an $S U(2,2)$-subgroup $G_{12}$ in G3. Namely, each $g_{12}$ in $G_{12}$ is composed (from blocks $A_{2}, B_{2}, C_{2}, D_{2}$ of the original matrix $g_{2}$ from $\left.G_{2}=S U(2,2)\right)$ as follows: $A_{2}$ is the upper principal minor in the 6 by 6 matrix $g_{3} ; D_{2}$ is the principal minor located at the 4 and 5 row-column intersections; one more (not a principal) minor $B_{2}$ is located on the intersection of rows (1 and 2) with columns (4 and 5); $C_{2}$ is located on the intersection of rows (4 and 5) with columns ( 1 and 2$)$. The remaining entries of the matrix $g_{12}$ are as follows: 1 (if on the main diagonal) and 0 (when off the main diagonal). A reader is now advised to have a look at Fig. A5. Subgroups $G_{13}$ and $G_{23}$ are defined quite similarly. The following statement can be easily verified. 
Proposition 1. The subgroup $G_{12}$ acts linear-fractionally on $D_{12}$; similarly: $G_{13}$ on $D_{13}$, and $G_{23}$ on $D_{23}$.

Remark 5. Actually, we can (canonically) define an (equivalent to linear-fractional) action of each (of the three possible) $G_{i j}$ on $D_{\text {sk. }}$. As an example, to define such an action of $G_{13}$ on $D_{12}$, we conjugate $G_{13}$ by the corresponding elementary matrix E. Now, if $g$ is from $G_{13}$ and $z$ is from $D_{12}$, then the result of the $g$-action on $z$ is, by definition, the result of the (linear-fractional) action of $E g E$ on $z$. From now and on, we assume that each $G_{i j}$ acts (in the way which has been just indicated) on any of $D_{\text {sk }}$.

Having the Remark 5 in mind, we define the color of an MLM-quark Dsk as Gij (which can be chosen from $G_{12}, G_{13}, G_{23}$ ). In other words, the color of an MLM-quark is defined by the choice of its ruling group (or, even more formally, the color (as a symbol) of an MLMquark is (the symbol of) its ruling group. It goes without saying that the ruling group also acts in the Hilbert space of wave functions of the quark in question (compare to what we have stated earlier, right before Remark 3). Clearly, there are three colors for quarks of the $U(3)$-level.

Let us now introduce the notion of a color for an arbitrary $U(n)$, with the integer $n$ not less than 3. Given an embedding of $\mathbf{D}=U(2)$ into $U(\mathrm{n})$, by $G_{\mathrm{ij}}$ we understand a certain (uniquely defined) $S U(2,2)$-subgroup in $G_{n}=S U(n, n)$. Namely, $G_{i j}$ consists of certain matrices $g_{\mathrm{n}}$, uniquely defined by four $n$ by $n$ blocks $A_{\mathrm{n}}, B_{\mathrm{n}}, C_{\mathrm{n}}, D_{\mathrm{n}}$. The latter four blocks are uniquely defined by the matrix $g_{2}$ (chosen arbitrarily) from $G_{2}=S U(2,2)$; in particular, $G_{\mathrm{ij}}$ is isomorphic to $S U(2,2)$ - see Proposition 2, below. To continue, $g_{2}$ is determined by its 2 by 2 blocks $A_{2}, B_{2}, C_{2}, D_{2}$. To define each $n$ by $n$ block for $g_{n}$, proceed as follows. For an arbitrary $n$ by $n$ matrix, $A_{\text {ij }}$ determines the corresponding 2 by 2 principal minor in it (it might again be helpful to check with our Fig. A5 ). We define $A_{\mathrm{n}}$ as follows: (1) $A_{2}$ is that very minor in $A_{\mathrm{n}}$; (2) any other entry in $A_{\mathrm{n}}$ is 1 (if on the principal diagonal) or it is zero (if it is off the principal diagonal) - compare to how $G_{12}$ was defined in our Section 2. The block $D_{\mathrm{n}}$ is defined quite similarly but with the help of $D_{2}$. The two remaining blocks, $B_{\mathrm{n}}$ and $C_{n}$, are defined (in terms of $B_{2}$ and $C_{2}$ ) slightly differently. Namely, each entry, which is off the corresponding 2 by 2 principal minor of the block, is zero. The following statement has been proven in [2].

Proposition 2. $G_{\mathrm{ij}}$ is a subgroup of $G_{\mathrm{n}} ; G_{\mathrm{ij}}$ is isomorphic to $\operatorname{SU}(2,2)$.

For each level $U(\mathrm{n})$, with $n>2$, the MLM-quark (of a certain flavor and color) is defined as an ordered triple $\left(\mathrm{D}_{\mathrm{pq}}, \mathrm{G}_{\mathrm{ij}}, m\right)$. Here $m$ is either 1 , or negative 1 (depending on whether we deal with a particle or with an anti-particle). The subgroup $D_{p q}$ in $U(n)$ determines flavor, while the subgroup $G_{i j}$ in $S U(n, n)$ determines color. An implicit part of this definition is a well-defined representation space, $p$-space $H$, which the proton's wave function belongs to. The ruling group $G_{\mathrm{ij}}$ acts in this $H$.

Here is another statement from [2]:

Proposition 3. The total number of colors at the $U(n)-l e v e l$ is $n(n-1) / 2$.

Returning to the $U(3)$-level, let us now present the following possible interpretation in terms of physics: when a proton (participating in highly inelastic scattering) finds itself in a $\mathrm{D}_{13}$-cell (and it stays there for a moment, name it a sunken proton), then its color may be one of $G_{12}, G_{23}, G_{13}$ but changing between them with huge speed, presumably. As stated in the above Table 1 , in $D_{13}$ these ruling subgroups generate electric charges 0,0 , negative 1 , in that order. It means that a quark $d$ has an average charge of $1 / 3$. Similarly, a $u$-quark has charge 2/3. Notice that (from now and on) we use sunken instead of sank (which appeared in [2]). As regards an electric charge generation by the ruling group, we provide more details in our subsection 6.2.

For the $U(4)$-level, consider the following

Table 2.

\begin{tabular}{ccccccc}
\hline $\mathbf{f} \backslash \mathbf{c}$ & $\mathbf{1 2}$ & $\mathbf{1 3}$ & $\mathbf{1 4}$ & $\mathbf{2 3}$ & $\mathbf{2 4}$ & $\mathbf{3 4}$ \\
\hline $\mathrm{u}: 12,34$ & 1 & 0 & 1 & 1 & 0 & 1 \\
$\mathrm{~d}: 13,24$ & 0 & -1 & 0 & 0 & -1 & 0 \\
\hline
\end{tabular}




\begin{tabular}{ccccccc}
\hline s: 14 & 0 & -1 & 0 & 0 & -1 & 0 \\
c: 23 & 1 & 0 & 1 & 1 & 0 & 1 \\
\hline
\end{tabular}

Again, applying the Han-Nambu scheme, we deduce 'correct' electric charges for MLM-quarks $u, d, c, s$ at this level. The total number of colors is 6 .

At the $U(5)$-level, there are 10 colors (see Table 3 ) which implies charges of $7 / 10$ for each of $u, c, t$ and negative 3/10 charges for each of $d, s, b$. Hence, at the level of $U(5)$ the SM-data and the MLM-conclusion (assuming the validity of the Han-Nambu scheme) are contradictory (as regards quarks' electric charges).

Remark 6. Seemingly, one might try to exploit this discrepancy while experimenting with the $\mathrm{e}^{+}-\mathrm{e}$ - annihilation. Frequently one plots the curve $R(s)=$ (total cross section to get hadrons) over (total cross section to get muon and anti-muon pair). Here $s$ is the square of the total energy in the center of inertia. The knowledge about that curve is fundamental for the current high-energy physics, see [21] (p. 269). Outside of the resonances, the curve is piece-wise constant: $R$ is 3 times the sum of $f^{2}$, where the summation goes for all quarks having a smaller mass than energy; here $f=f(q)$ is an electric charge of the corresponding quark $q$. However, one has to deduce the MLM-analogue of this formula, since the number of colors is now level-dependent. Nevertheless (even without exact knowledge of this formula), we can guess (assuming the validity of the Han-Nambu scheme) that on the $U(5)$ level the function $R$ behaves somewhat differently if compared with its behavior in levels $U(3), U(4)$, and $U(6)$. Notice that in [2] (Section 6) the corresponding numerical corrections have been suggested (by A. L.) too hastily. As we have just indicated the question seems to be more complicated and it still waits for its solution.

Table 3.

\begin{tabular}{ccccccccccc}
\hline $\mathbf{f} \backslash \mathbf{c}$ & $\mathbf{1 2}$ & $\mathbf{1 3}$ & $\mathbf{1 4}$ & $\mathbf{1 5}$ & $\mathbf{2 3}$ & $\mathbf{2 4}$ & $\mathbf{2 5}$ & $\mathbf{3 4}$ & $\mathbf{3 5}$ & $\mathbf{4 5}$ \\
\hline u: 12,45 & 1 & 0 & 1 & 1 & 0 & 1 & 1 & 1 & 0 & 1 \\
d: 13,35 & 0 & -1 & 0 & 0 & -1 & 0 & 0 & 0 & -1 & 0 \\
s: 14,25 & 0 & -1 & 0 & 0 & -1 & 0 & 0 & 0 & -1 & 0 \\
c: 12,45 & 1 & 0 & 1 & 1 & 0 & 1 & 1 & 1 & 0 & 1 \\
b: 14,25 & 0 & -1 & 0 & 0 & -1 & 0 & 0 & 0 & -1 & 0 \\
t: 12,45 & 1 & 0 & 1 & 1 & 0 & 1 & 1 & 1 & 0 & 1 \\
\hline
\end{tabular}

At the $U(6)$-level the number of colors is 15, see Table 4 . The MLM-quarks on this level get three new flavors. The electric charges of the new quarks are as follows: $f(4 ; 1,6)$ $=f(4 ; 3,4)=2 / 3, f(4 ; 2,5)=-1 / 3$. The charges of other MLM-quarks on this level are the same as of the corresponding SM-quarks.

Table 4 .

\begin{tabular}{cccccccccccccccc}
\hline $\mathbf{f} \backslash \mathbf{c}$ & $\mathbf{1 2}$ & $\mathbf{1 3}$ & $\mathbf{1 4}$ & $\mathbf{1 5}$ & $\mathbf{1 6}$ & $\mathbf{2 3}$ & $\mathbf{2 4}$ & $\mathbf{2 5}$ & $\mathbf{2 6}$ & $\mathbf{3 4}$ & $\mathbf{3 5}$ & $\mathbf{3 6}$ & $\mathbf{4 5}$ & $\mathbf{4 6}$ & $\mathbf{5 6}$ \\
\hline $\mathrm{u}: 12,45$ & 1 & 0 & 1 & 1 & 0 & 1 & 0 & 1 & 1 & 1 & 0 & 1 & 1 & 0 & 1 \\
$\mathrm{~d}: 13,35$ & 0 & -1 & 0 & 0 & -1 & 0 & -1 & 0 & 0 & 0 & -1 & 0 & 0 & -1 & 0 \\
$\mathrm{~s}: 14,25$ & 0 & -1 & 0 & 0 & -1 & 0 & -1 & 0 & 0 & 0 & -1 & 0 & 0 & -1 & 0 \\
$\mathrm{c}: 12,45$ & 1 & 0 & 1 & 1 & 0 & 1 & 0 & 1 & 1 & 1 & 0 & 1 & 1 & 0 & 1 \\
$\mathrm{~b}: 14,25$ & 0 & -1 & 0 & 0 & -1 & 0 & -1 & 0 & 0 & 0 & -1 & 0 & 0 & -1 & 0 \\
$\mathrm{t}: 12,45$ & 1 & 0 & 1 & 1 & 0 & 1 & 0 & 1 & 1 & 1 & 0 & 1 & 1 & 0 & 1 \\
$\mathrm{q}(4 ; 1 ; 6)$ & 1 & 0 & 1 & 1 & 0 & 1 & 0 & 1 & 1 & 1 & 0 & 1 & 1 & 0 & 1 \\
$\mathrm{q}(4 ; 2 ; 5)$ & 0 & -1 & 0 & 0 & -1 & 0 & -1 & 0 & 0 & 0 & -1 & 0 & 0 & -1 & 0 \\
$\mathrm{q}(4 ; 3 ; 4)$ & 1 & 0 & 1 & 1 & 0 & 1 & 0 & 1 & 1 & 1 & 0 & 1 & 1 & 0 & 1 \\
\hline
\end{tabular}

5. The Multi-Level Model has infinitely many quarks' and leptons' generations 
As should be already clear, the MLM's main feature is the sequence of levels: $U(2)$, $U(3), U(4)$, etc. In our Scheme 1, below, we denote MLM-quarks by $p_{\mathrm{ij}}$ rather than by the SM-symbols, as before. The reason for doing so is as follows.

\subsection{A 'sunken vs captured' MLM-proton}

In our Section 4 (right after Proposition 3) we have provided more details on 'a quark as a sunken proton' approach (as originally articulated in [2] and recalled in the beginning of our Section 6, below). Namely, any subgroup $G_{\mathrm{ij}}$ of $S U(\mathrm{n}, \mathrm{n})$ induces the color charge, of $1,-1$, or 0 in each 'cell' $D_{\text {sk }}$ of the $U(n)$-level. In the current Section 5 we discuss MLMquarks (and MLM-leptons which can be associated with them) as stable ingredients of 'matter at deeper (than $U(2)$ ) levels'. Such stability presumes that A PROTON STAYS AT THE Dsk-CELL NOT JUST FOR A TINY MOMENT (which could have happened as the result of its participating in highly inelastic scattering - see the portion of our Section 4, between Proposition 3 and Remark 6) BUT FOR AN OBSERVABLE (and even theoretically unlimited, if there is no influence by another interaction) TIME INTERVAL - name it a CAPTURED PROTON. Let us postulate that such A CAPTURE MAY HAPPEN ONLY IF the ruling group $G_{i j}$ 'matches' the cell $D_{s k}: i=s$ and $j=k$. In particular, such a hadron $p_{i j}$ has electric charge of 1 or of negative 1 .

\subsection{Fermion triples at deeper levels and where to search for new quarks and leptons}

Having in mind our Remarks 1 and 2, it is natural to place MLM-fermions of spin 1/2 into levels and to combine them into triples as follows:

\section{Scheme 1.}

\begin{tabular}{|c|c|}
\hline $\begin{array}{l}U(2)- \\
\text { level: }\end{array}$ & proton $p$ - electronic antineutrino - electron $e$. \\
\hline$U(3)-$ & quark $p_{12}\left(\right.$ or $\left.p_{23}\right)-$ muonic antineutrino - muon $\mu$, \\
\hline level: & quark $p_{13}-$ muonic neutrino - anti- $\mu$. \\
\hline$U(4)-$ & quark $p_{12}\left(\right.$ or $\left.p_{34}\right)-$ muonic antineutrino $-\mu$, \\
\hline level: & quark $p_{13}\left(\right.$ or $\left.p_{24}\right)-$ muonic neutrino - anti- $\mu$, \\
\hline & quark $p_{23}-($ anti - tau neutrino $)-$ tau particle $\tau$, \\
\hline & quark $p_{14}-$ tau neutrino - anti- $\tau$. \\
\hline$U(5)-$ & quark $p_{12}\left(\right.$ or $\left.p_{45}\right)-$ muonic antineutrino $-\mu$, \\
\hline \multirow[t]{5}{*}{ level: } & quark $p_{13}\left(\right.$ or $\left.p_{24}\right)-$ muonic neutrino - anti- $\mu$, \\
\hline & quark $p_{23}\left(\right.$ or $\left.p_{35}\right)-($ anti - tau neutrino $)-\tau$, \\
\hline & quark $p_{14}\left(\right.$ or $\left.p_{25}\right)-$ tau neutrino - anti- $\tau$, \\
\hline & quark $p_{24}-4^{\text {th }}$ generation neutrino $-4^{\text {th }}$ generation positron, \\
\hline & quark $p_{15}-4^{\text {th }}$ generation antineutrino $-4^{\text {th }}$ generation electron. \\
\hline
\end{tabular}

The main letter $p$ in the (above used) notation, reminds that each MLM-quark $p_{\text {ij }}$ originates from a proton $p$, essentially. Besides, $p_{\mathrm{ij}}$ stands for a captured proton (as postulated above). Also, it now makes sense to reproduce from [9] (Section 6) Scheme 2 (see below) which is an 'old version' of Scheme 1. Clearly, a physicist (not enough informed about our MLM-setting) might think of the Scheme 2 as of an awkward one (if not contradictory): how an SM-quark (having fractional electric charge) can be associated with a lepton (having an integer charge)? In that case, we address him/her to the above Scheme 1.

Scheme 2. (an old version of Scheme 1). 


\begin{tabular}{|c|c|}
\hline $\begin{array}{l}U(2)- \\
\text { level: }\end{array}$ & proton - electronic antineutrino - electron \\
\hline $\begin{array}{l}U(3)- \\
\text { level: }\end{array}$ & $\begin{array}{l}\left.\text { quark } u \text { (represented by either } \mathrm{D}_{12} \text { or by } \mathrm{D}_{23}\right) \text { - muonic antineutrino - } \\
\text { muon } \mu \text {, } \\
\left.\text { quark } d \text { (represented by } \mathrm{D}_{13}\right) \text { - muonic neutrino - anti- } \mu\end{array}$ \\
\hline$U(4)-$ & quark $u$ (represented by either $\mathrm{D}_{12}$ or by $\mathrm{D}_{34}$ ) - muonic antineutrino $-\mu$, \\
\hline level: & $\begin{array}{l}\left.\text { quark } d \text { (represented by either } \mathrm{D}_{13} \text { or by } \mathrm{D}_{24}\right)- \text { muonic neutrino }- \text { anti- } \mu \text {, } \\
\text { quark } c \text { (represented by } \mathrm{D}_{23} \text { ) - anti - tau neutrino - tau particle } \tau \text {, } \\
\text { quark } s\left(\text { represented by } \mathrm{D}_{14}\right)-\text { tau neutrino }- \text { anti- } \tau\end{array}$ \\
\hline$U(5)-$ & quark $u$ (represented by either $\mathrm{D}_{12}$ or by $\mathrm{D}_{45}$ ) - muonic antineutrino $-\mu$, \\
\hline level: & $\begin{array}{l}\left.\text { quark } d \text { (represented by either } \mathrm{D}_{13} \text { or by } \mathrm{D}_{24}\right)- \text { muonic neutrino }- \text { anti- } \mu \text {, } \\
\left.\text { quark } c \text { (represented by either } \mathrm{D}_{23} \text { or by } \mathrm{D}_{35}\right)-(\text { anti }- \text { tau neutrino) }-\tau \text {, } \\
\left.\text { quark } s \text { (represented by either } \mathrm{D}_{14} \text { or by } \mathrm{D}_{25}\right)- \text { tau neutrino }- \text { anti- } \tau \text {, } \\
\left.\text { quark } b \text { (represented by } \mathrm{D}_{24}\right)-4^{\text {th }} \text { generation neutrino }-4^{\text {th }} \text { generation } \\
\text { positron, } \\
\left.\text { quark } t \text { (represented by } \mathrm{D}_{15}\right)-4^{\text {th }} \text { generation anti-neutrino }-4^{\text {th }} \\
\text { generation electron }\end{array}$ \\
\hline
\end{tabular}

In the lowest two lines of (each of the two) schemes, we have entered two leptons (of the $4^{\text {th }}$ generation) which are not yet experimentally detected. Each of the above triples (like 'quark $u-$ muonic antineutrino $-\mu$ ' or 'quark $c-$ tau antineutrino $-\tau^{\prime}$ ') is entered on the basis of the 'proton - electronic antineutrino - electron' composition series, see our Remarks 1 and 2. Also, notice that a lepton of generation $k$ is always associated with a quark of generation $k-1$. This property originates from the following convention: by 'quark of generation $0^{\prime}$ we mean a proton. Both the $U(2)-$, and the $U(3)$-part of the above data is illustrated in Figure A4 (with more explanation added there).

The following observation finalizes the validation of our Section 5 title: one can similarly proceed with levels $U(6), U(7)$, etc. In particular, Figure A3 illustrates the $U(6)$-level and indicates 'where' to search for (three) quarks of the $4^{\text {th }}$ generation.

\subsection{Why are different generations' leptons distinct particles?}

One of the 'puzzles' of the Standard Model reads as follows: "Why electron $e$, muon $\mu$ and tau lepton $\tau$ are distinct particles?" Here is our reply (we give it as one of the MLMconclusions and we use the terminology developed in our Section 2):

Remark 7. Within the SM (when the Wigner method is applied), the above three leptons are described by one and the same pair $(H, G)$, where $H$ is a Hilbert space of wave functions and $G$ is the $r$-group (being the 10-dimensional Poincare one). Assuming the validity of the MLM, our MLM-leptons have different spaces $\left(H_{1}, H_{2}, H_{3}\right)$ of wave functions and different $r$-groups: $G_{1}, G_{2}, G_{3}$ (these groups are 15-dimensional). Namely, the three supports of wave functions are pair-wise distinct since they are quotient spaces produced by factoring at three different levels (as it has been earlier mentioned, in such a situation the Wigner-Segal method is necessarily involved). As regards the groups $G_{1}, G_{2}$, and $G_{3}$, the first consists of 4 by 4 matrices, the second - of 6 by 6 matrices, and the third - of 8 by 8 ones. Also, these $G_{1}, G_{2}, G_{3}$ operate at different levels: at $U(2)$, at $U(3)$, and at $U(4)$, respectively. Having all the above in mind, it seems as of not much of a surprise that the three MLM-leptons are observed as distinct particles.

Remark 8. Quite similarly (within the MLM), it can be explained why any two neutrinos (of two different SM-generations) are detected as two distinct particles.

Remark 9. We would like to interpret our list of fermions' triples in levels $U(2)$ through $U(5)$ (presented earlier in this Section) as a hint on how the matter at deeper 
MLM-levels might be structured. Maybe the detection of hadron jets is indicative of such a structure (which might be locally damaged during a highly energetic interaction)? These types of questions are in line with findings of [22, 23]. Namely, [22] concludes about quark-gluon plasma as of an extremely dense media (at least, as studied in central $\mathrm{Pb}-\mathrm{Pb}$ collisions). Certain parts of [23] describe 'quark matter'. In particular, it is concluded that the 'hadron-quark pasta' phase exists in very massive neutron stars, whose rotational frequencies are less than around $300 \mathrm{~Hz}$. All other stars are not dense enough to trigger quark deconfinement in their cores. Recall in this regard that the $U(2)$-level is a chronometric model of 'our' space-time. That is why the presented combination of fermions' triples in levels $U(3), U(4)$, and $U(5)$ (see Scheme 1) can be interpreted as the way of how 'matter' at these $(=$ 'deeper') levels might be structured. Notice that the above Scheme 1 presumes integer charges (that is 1, or negative 1) of stable MLM-quarks. Otherwise, as an example, the following triple (quark $d$ - muonic neutrino - antimuon) would not be of total zero charge; then how could we view it as the 'U(3)-level anti-neutron'?

\section{Discussion}

Chronometric fermions of spin $1 / 2$ are introduced via the spannor representation (see the portion of our text right before Theorem 2 in Section 2). In [10] it has been proven that the corresponding composition series is a 'three-step' one. Its lower and upper factors are interpreted as the proton and the electron. Since factoring here is inescapable, this procedure (of constructing elementary particles) is not described in terms of the Wigner method. In [5] it was suggested to name such an approach (which the above three-step composition series is a specific feature of) as the Wigner-Segal method. In [2] it has been stated that as a basis for the MLM, the Segal's compact cosmos D can be exploited as well as the sequence of canonical (that is, corresponding to principal minors of appropriate matrices) group embeddings: $\mathrm{U}(2)$ into $\mathrm{U}(3), \mathrm{U}(2)$ into $\mathrm{U}(4)$, etc. These groups were called the levels (of matter): $U(2)$ - the $0^{\text {th }} \quad$ (that is, our mundane world), $U(3)$ - the $1^{\text {st }}, U(4)$ - the $2^{\text {nd }}$, etc. Such a convention matches the SM-quarks' generations list. An opinion has been expressed [2], that "the MLM is the only known construct where such notions as flavor and color are defined mathematically". Currently, the following seems to be an adequate description of the 'MLM versus SM' case: MLM is the symbiosis of Segal's Chronometry with SM.

As regards another statement from [2] ("In MLM, a quark can be interpreted as a 'sunken' proton: during the beginning of the reaction process, the proton 'is pushed' into a 'deeper' level."), our current paper provides (by presenting findings from several publications in one place here) a much more detailed foundation for such a conjecture. Unfortunately, we are not yet in a position to better support another claim of [2] ("At each level, a gluon can be interpreted as a colored and flavored photon."), since in order to do that, we have to start with a double-check of the bosonic sector findings by Segal. Recall that in [10] such a double-check has been performed for the fermionic sector. Nevertheless, even at this point in time it seems appropriate to mention the following chronometric findings: The key bosons (photon $\gamma, \mathrm{W}$-boson, Z-boson) have been mathematically detected [3].

Formally, the MLM (as applied to quarks and leptons) is presented as a rigorous mathematical construct. Since it is quite broad and involved, it allows to formulate (and, in some cases, it even calls for) logically reasonable conjectures and avenues of further research. Let us list some of them (as we have just mentioned, if bosons had been included/added as the objects of MLM-inspection, then such a list would have been broader). In each of the subsections (below), there is very little, as regards precise computations, since our exposition here merely INDICATES 'MLM-possibilities' which, presumably, can be further exploited in order to perform the research in question.

\subsection{MLM-mathematics of weak and strong interactions}

As regards both fermions and bosons, one interesting future research direction can be highlighted as follows: Can weak interactions be mathematically described as the MLM- 
vertical ones? To better understand the meaning of such a claim, the reader is invited to go back to our Remark 2 in Section 2. This was an example of a 'vertical' force binding the three particles (proton, anti-neutrino, and electron) together into a neutron. To mathematically decompose such a neutron into its ingredients, the factoring has to be applied. While by the MLM-horizontal, one should mean a 'tangential' (to a $U(\mathrm{n})$-level in question) force between quarks - the strong force, essentially (it is advised to have a look at those levels' content: Fig. A3). Or, which is even more mathematically precise, this force is between such a pair of particles (in one and the same $U(n)$-level) that each particle's space of states is an invariant 'massive subspace' of the corresponding representation (which is a spannor representation, essentially - see our Remark 1 in Section 2) of its ruling group. A generic example of such a subspace was $F_{p}$ from Remark 1.

\subsection{Chronometry and MLM on the notion of electric charge}

On the basis of our (chronometric) Remark 2 (in Section 2), and on the suggested MLM- approach to the values of SM-quarks' electric charges (see in our Section 4 the portion of the text which goes after Proposition 3), the following seems to be logically noncontradictory:

Conjecture 1. The electric charges of the proton and of the electron (viewed either 'inside' neutron, or separately) originate as the result of the corresponding action (in their Hilbert spaces $F_{\mathrm{p}}$ and $F_{\mathrm{e}}$ ) of the ruling group $G$ (represented, essentially, by $S U(2,2)$ ).

Hopefully, such a (to a certain extent, philosophical) view might serve as an answer (preliminary, at least) to the question "What are the origins of electric charge?" According to the SM (as well as to the MLM), there are color charges, too. In our Section 4 the color charges were introduced via the 'MLM-modification' of the Han-Nambu scheme. The electric charges of the SM-quarks are fractional. As based on our Section 4 findings, it is possible to interpret the chronometric proton's electric charge as a special case of the MLM-quarks' color charges: in the $U(2)$-level there is just one ruling group for the proton which means that there is just one color. We interpret this color charge as the electric charge of the chronometric proton.

An MLM-quark is a MATHEMATICAL notion. At each level, it is interpreted (in terms of Physics) as a 'sunken proton' (or a 'proton in a cell'). The color of the MLM-quark is defined by its ruling group. In the $U(3)$-level, there are three $\left(G_{12}, G_{13}, G_{23}\right)$ possible ruling groups, each is a subgroup of $S U(3,3)$. Hence, there are three color charges in the $U(3)$ level (the 'value' of each charge is 1 , see Table 1). And so on: in any level $U(\mathrm{n})$ each color charge is 'generated' by the corresponding subgroup (isomorphic to $S U(2,2)$ ). The electric charge of each sunken MLM-quark was defined as a statistical average of corresponding color charges (see a more detailed description which starts right after Table 1 in Section 4) - compare to the charge of a captured MLM-quark as described in our subsection 5.1.

The total number of colors (in a given MLM-level) is level-dependent (see Theorem 3 in Section 3). In the $\boldsymbol{U}(5)-$ level, the electric charge values of MLM-quarks are (slightly) different from those of the corresponding SM-quarks.

\subsection{On the nucleon's mass and shape (the case of a chronometric proton)}

In the beginning of our Section 2 we have spoken on the interplay between chronometric and relativistic notions. As regards the notion of mass (of an elementary particle), our guiding 'tools' are the ones suggested by Segal (see [3] and references therein). In particular, [3] (p. 996) provides the expression for the relativistic mass operator. In view of the very MLM-structure, it seems reasonable to start with the case of the (chronometric) proton $p$. In particular, it is necessary to search for most suitable (in view of what Segal says on p.996 of [3]) p-states. Here we present an outcome of one small step forward in the performance of such a search.

Recall from our Section 2 that we there view the proton's wave functions as defined on the Minkowski space-time $M$. In [10] $M$ is represented as a certain set of 2 by 2 matrices, let us use $\mathrm{h}$ to denote an element in $M$. It follows from [10] (equation (20)) that each 
value of the wave function $\mathrm{L}$ at $\mathrm{h}$ is a vector in $\mathrm{C}^{2}$. The latter is a complex 2-dimensional linear space. Using equation (15) of [10], we have:

$$
\mathrm{L}(\mathrm{h})=\mathrm{SAv} \text {, }
$$

and nowhere vanishing

$$
\mathrm{S}=\{\operatorname{det}[(\mathrm{h}-\mathrm{w}) / 2 \mathrm{i}]\}^{-2} \text {. }
$$

In these two expressions a 2 by 2 matrix $w$ (for which $h-w$ is non-singular for all $h$ ), and a vector $\mathrm{v}$ from $\mathrm{C}^{2}$, serve as parameters. In other words, to choose a specific wave function $\mathrm{L}$, we have to specify $\mathrm{w}$ and $\mathrm{v}$, first. The expression for the 2 by 2 matrix $\mathrm{A}$ is more involved: these and other details are available in [24]. In this [24], each of the parameters in (3), (4) has been specified and the real-valued function $f(x, y)$ has been composed as the hermitian square of $\mathrm{L}(\mathrm{h})$ in $\mathrm{C}^{2}$. Here $\mathrm{x}$ and $\mathrm{y}$ are the 'usual' coordinates on the plane, while the remaining two coordinates (time $\mathrm{t}$ and space-coordinate $\mathrm{z}$ ) are chosen as 0 . In other words, it is the case of a 'toy model', a very simple first try to understand what kind of functions might be obtained, in the proton's Hilbert space $F_{\mathrm{p}}$. The findings of such a try are graphically presented: Figures B1, B2, and B3. Clearly, it is important to move from this toy example to a more realistic one where $\mathrm{f}$ depends on all four variables (notice that, obviously, $\mathrm{f}(\mathrm{x}, \mathrm{y}, \mathrm{z}, \mathrm{t})$ is everywhere positive).

In particular, it seems reasonable to look for a wave function (3) with the following properties:

A) sections of $f(x, y, z, t)$ by 3-planes $t=$ const are spherically symmetric (or, at least, they are close to being spherically symmetric);

B) as $t$ changes, these sections change rather slowly;

C) the value $f(x, y, z, t=$ const $)$ is very close to 0 as soon as $(x, y, z)$ is far enough from the center of our proton (we are thus able to speak of the proton's radius).

If the value (as a function of time) of the mass operator at such a state is almost a constant, then we can set this value to be the proton's mass. In what follows, we pretend that such a realistic wave function has been already detected. In that case, it is possible to relate our preliminary analytic findings in the proton's space $F_{\mathrm{p}}$ to certain attempts of research, dedicated to the radius of a proton or, more broadly, to the shape of nucleons: [25 -27].

As it is clearly seen on Fig. B1, $(-1,0)$ is the center of our proton. The function $f(x, y)$ has a local minimum there. As analytic calculations [24] show, the graph of $f(x, y)$ is rotationally symmetric. The axis of this symmetry, the straight line $\mathrm{N}$ (passing through $(-1,0)$ ) is orthogonal to the plane of coordinates $x, y$ and $N$ passes through $(-1,0)$. On Fig. B2 we see that the highest points of the graph form a circle. This circle (or, rather a 2-sphere, for a more realistic example) is "where a strong repulsive pressure near the centre of the proton (up to $0.6 \mathrm{fm}$ ) switches to a binding pressure at greater distances" [25]. As Fig. B3 shows, our condition C), from above, is close to being satisfied.

\subsection{The SM states that a proton consists of three quarks. What does MLM say?}

In Section 2 (closer to its end) we have described the MLM-interpretation of the proton's uud-quark structure, the latter being one of the basic SM-tenets. The Multi-Level Model claims that the proton is indestructible since its Hilbert space of states, $F_{\mathrm{p}}$, carries the irreducible unitary representation of $G=S U(2,2)$. Instead of interpreting $u, u, d$, as particles, the MLM explains the detection of three centers of electron-proton scattering as follows: MLM speaks of three 'channels' which the proton 'chooses' in order to avoid destruction when bombarded (by electrons, say). These channels can be labeled by symbols $D_{i j}$ of corresponding cells (see Definition 1 in Section 2). Having in mind our contemplation (see Section 5) on the structure of matter at deeper, than $U(2)$, levels, we can now put into play one more possible feature of such a highly inelastic electron-proton scattering. It looks like a reasonable physical assumption that the corresponding cell ( $\mathrm{D}_{12}$, say, at the $U(3)$-level) is already 'occupied' (by a proton $p$, compare to the notation $p_{12}$ which we have 
introduced in Section 5, between Scheme 1 and Scheme 2). The former 'D 12 -occupant' is then pushed to an even deeper level, $U(4)$, for a moment. Our proton, now being in $\mathrm{D}_{12}$, repels the electron elastically (that is, electron bounces off the proton when the latter is in the $\mathrm{D}_{12}$ cell), and then our proton is pushed (by the deeper matter's repulsive pressure) back to the $U(2)$-level.

Remark 10. An alternative view on the three centers in the electron-proton scattering has been recently expressed by M. Dulin (Novosibirsk). According to it, the proton exists, simultaneously, in three states. Such a property expresses itself as if there are three scattering centers.

\section{Conclusions}

The main goal of the current review is to put together mathematical arguments and experimental data in order to support the claim that the Multi-Level Model can be thought of as the symbiosis of Chronometry with the Standard Model. Above, we mostly have been using the setting of Segal's Chronometry in its compact form, $\mathrm{D}=U(2)$. There is a mathematically well-developed [12] (Sections 3.1 and 7) way of transition to an astronomically more adequate setting, the direct product of the 'time line' with $S U(2)$, where $S U(2)$ is for a spherical 3-space with its radius $R$ being a conformal invariant [28]. Segal names this $\mathrm{R}$ as the "radius of the universe."

As Segal puts it [3], "...the chronometric physics is fundamental theory that derives from very general considerations of causality, stability, and symmetry. As such, it is naturally slightly abstract, and its empirical implications require development, which like those of special relativity and quantum mechanics may initially appear contradictory of accepted doctrine... Relativistic quantum theory appears as the limiting case of chronometric theory as $\mathrm{R}$ tends to infinity, in a sense similar to that in which classical physics appears as the limit (as the Planck's constant tends to 0) of quantum theory, and nonrelativistic physics appears as the limit (as the speed of light $c$ goes to infinity) of relativistic physics."

If, by now, we have been able to justify the title of our review, then the above characteristics of Chronometry are applicable to the Multi-Level Model, as a whole. Also, we would like to indicate that the MLM-approach to quarks and leptons is a very economic one: essentially, the number of stable elementary fermions of spin $1 / 2$ has been reduced to just three (the proton, the electron, the electronic neutrino and their anti-particles). Hopefully, the MLM-view that a proton is indestructible, will, eventually, be accepted by the mainstream Physics. Combined with the MLM-approach $(U(\mathrm{n})$-levels being the key wording here) to matter structuring, such a radical view on the proton makes it easy to solve the SM-puzzle of quark and lepton generations (as done in our Section 5).

Since our chronometric proton has no constituents, it is questionable whether there is an MLM-need for colors, per se. In our current presentation we stay with colors exactly as they have been mathematically introduced in [1, 2]. However, we have now highlighted the notion of the ruling group (which 'creates' color): Definition 2 in Section 2 . The number of colors is level-dependent (see formulas (1), (2) in Section 3). In Scheme 1, and in Fig. A3 we have indicated 'where' to search for new quarks and leptons.

The only original part of the current paper is the subsection 6.3, above. We suggest a wave function based approach to study certain properties of the proton (like mass, shape and inner pressure which have been recently researched in several publications).

Seemingly, only further theoretical research (and check against experimental data) will decide which other claims/conjectures/approaches (some outlined, some just mentioned) of our review may significantly contribute to the current trends in the Physics of Particles - while the remaining ones would be thought of as having merely mathematical value.

Conflicts of Interest: The authors declare no conflict of interest. 
Funding: The research by A. Levichev was partly funded by the Program of fundamental scientific research of the SB RAS No I.1.2., project No 0314-2019-0006.

Institutional Review Board Statement: Not applicable.

Informed Consent Statement: Not applicable.

Data Availability Statement: Not applicable.

\section{Appendix A}

Figure A1.

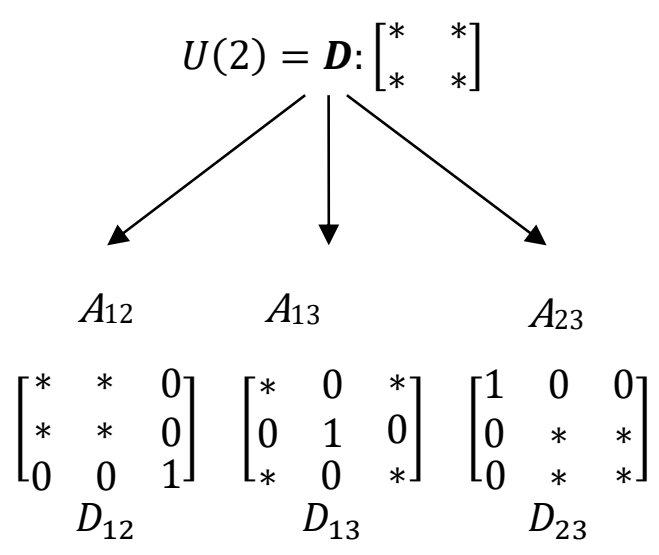

$D_{12}$, quark $u ; D_{13}$, quark $d ; D_{23}$, quark $u$ - subgroups in $U(3)$ isomorphic to $U(2)$.

$$
\begin{gathered}
P_{2}:\left[\begin{array}{ll}
z_{1} & z_{2} \\
z_{3} & z_{4}
\end{array}\right] \rightarrow\left[\begin{array}{ll}
z_{4} & z_{2} \\
z_{3} & z_{1}
\end{array}\right] ; P_{3}:\left[\begin{array}{lll}
a & b & c \\
d & e & f \\
g & h & i
\end{array}\right] \rightarrow\left[\begin{array}{lll}
i & f & c \\
h & e & b \\
g & d & a
\end{array}\right] . \\
A_{23}=P_{3} \circ A_{12} \circ P_{2}, \quad P_{3}\left(D_{12}\right)=D_{23} \\
P_{3}\left(D_{23}\right)=D_{12}, \quad P_{3}\left(D_{13}\right)=D_{13},
\end{gathered}
$$

Figure A2.

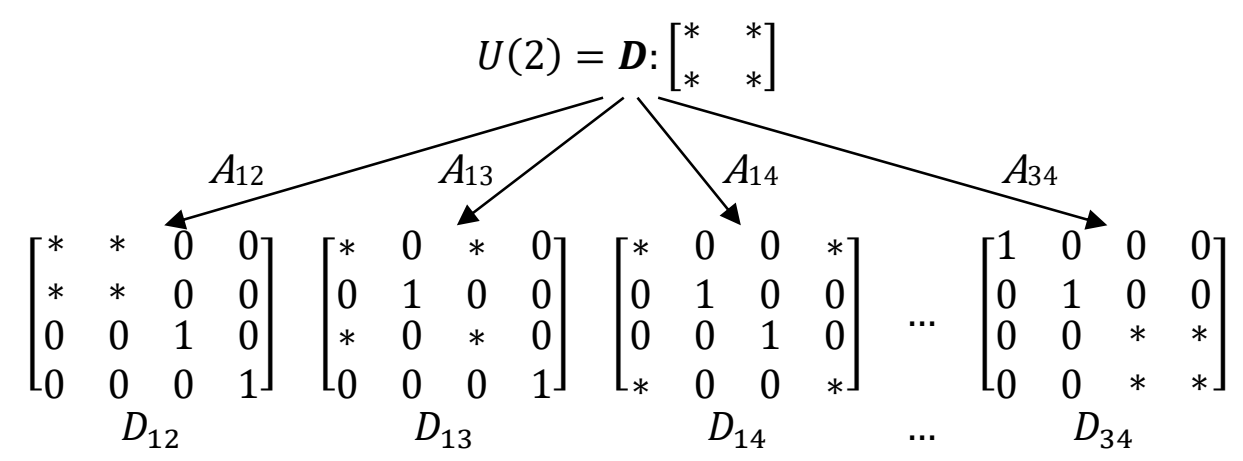

$A_{24}=P_{4} \circ A_{13} \circ P_{2}, \quad A_{34}=P_{4} \circ A_{12} \circ P_{2}, \quad P_{4}\left(D_{13}\right)=D_{24}, \quad P_{4}\left(D_{24}\right)=D_{13}$,

$P_{4}\left(D_{12}\right)=D_{34}, \quad P_{4}\left(D_{34}\right)=D_{12}$,

$P_{4}\left(D_{14}\right)=D_{14}-$ quark $s ; \quad P_{4}\left(D_{23}\right)=D_{23}-$ quark $c$.

Figure A3.

$U(6):\left\{\begin{array}{ccccc}D_{12}^{u} & D_{13}^{d} & D_{14}^{s} & D_{15}^{t} & D_{16}^{q(4 ; 1,6)} \\ & & & & 2 / 3 \\ D_{23}^{c} & D_{24}^{b} & D_{25}^{q(4 ; 2,5)} & D_{26}^{t} & D_{34}^{q(4 ; 3,4)} \\ & & -1 / 3 & & 2 / 3 \\ D_{35}^{b} & D_{36}^{s} & D_{45}^{c} & D_{46}^{d} & D_{56}^{u}\end{array}\right.$ 
$U(5):\left\{\begin{array}{ccccc}D_{12}^{u} & D_{13}^{d} & D_{14}^{s} & D_{15}^{t} & D_{23}^{c} \\ 2 / 3 & -1 / 3 & -1 / 3 & 2 / 3 & 2 / 3 \\ D_{24}^{b} & D_{25}^{s} & D_{34}^{c} & D_{35}^{d} & D_{45}^{u} \\ -1 / 3 & -1 / 3 & 2 / 3 & -1 / 3 & 2 / 3\end{array}\right.$

$U(4): \begin{array}{cccccc}D_{12}^{u} & D_{13}^{d} & D_{23}^{c} & D_{24}^{d} & D_{34}^{u} & D_{14}^{s} \\ 2 / 3 & -1 / 3 & 2 / 3 & -1 / 3 & 2 / 3 & -1 / 3\end{array}$

$U(3): \begin{array}{rrr}D_{12}^{u} & D_{23}^{u} & D_{13}^{d} \\ 2 / 3 & 2 / 3 & -1 / 3\end{array}$

The electric charges (above) are entered in accordance with the SM. Please compare with our Section 4 where the Han-Nambu scheme has been applied. In that case, the charges of the $U(5)$-quarks would be different (see the part of the text right before our Remark 6).

Figure A4.
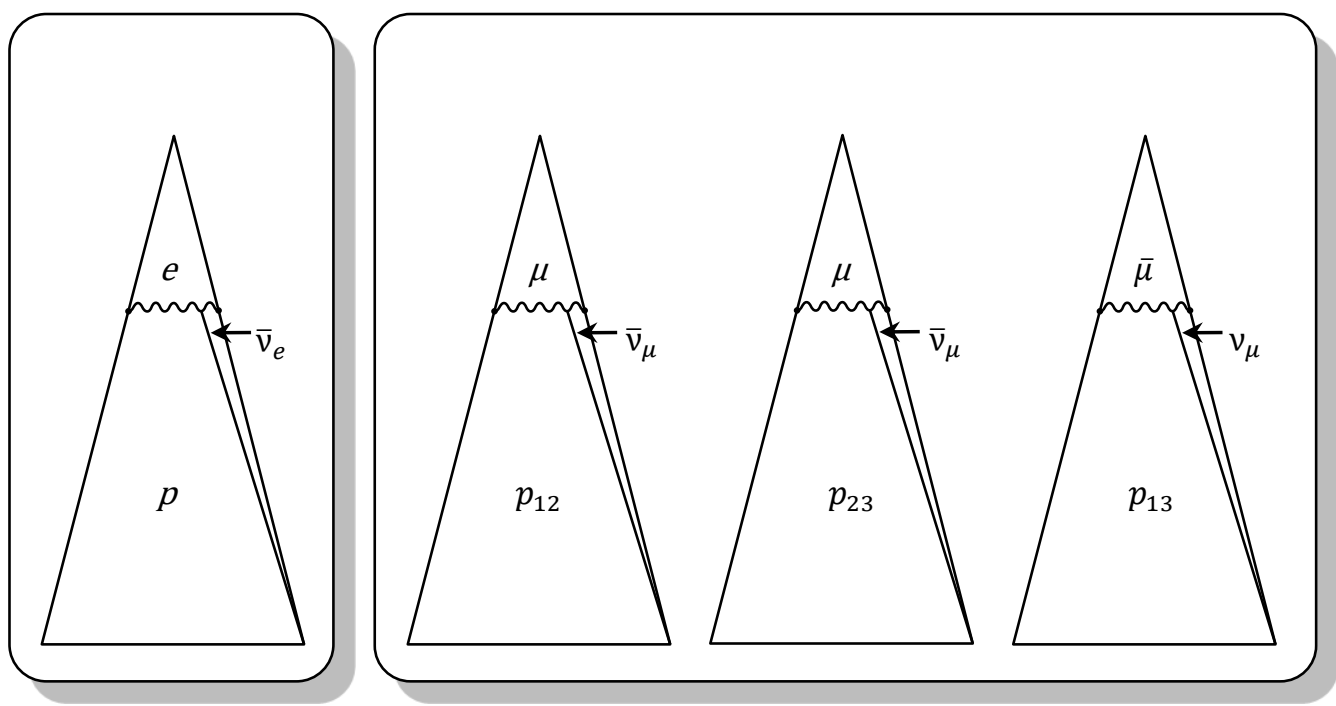

The left portion of Fig. A4 is for a neutron as consisting of the proton $p$, of an electron $e$, and of an electronic anti-neutrino. Different areas (for each the three particles) mean to indicate their different masses. The proton and neutrino are placed as 'equal' (in terms of the composition series - see our Remark 2 for more details) since each (of the two) representation subspace is invariant. Notice that there is also a significant mathematical difference between the two representation spaces. It is expressed in terms of the so called Gelfand-Kirillov dimension, and it allows to distinguish between the proton and the neutrino (see [10] for further details on that). There is no invariant subspace for the electron which is indicated by the wavy line. Additionally, this wavy line indicates the presence of the weak force which (together with electromagnetism) binds the three particles together, into a neutron. $5)$.

The right portion of Fig. A4 is for the $U(3)$-level (as discussed in Remark 9 of Section

Figure A5. 


$$
g_{12}=\left[\begin{array}{llll}
A & \mathbf{0} & B & \mathbf{0} \\
\mathbf{0} & 1 & \mathbf{0} & 0 \\
C & \mathbf{0} & D & \mathbf{0} \\
\mathbf{0} & 0 & \mathbf{0} & 1
\end{array}\right], g_{13}=\left[\begin{array}{ccc:ccc}
A_{1} & 0 & A_{2} & B_{1} & 0 & B_{2} \\
0 & 1 & 0 & 0 & 0 & 0 \\
A_{3} & 0 & A_{4} & B_{3} & 0 & B_{4} \\
\hdashline \bar{C}_{1} & 0 & C_{2}^{-} & D_{1}^{-} & 0 & D_{2} \\
0 & 0 & 0 & 0 & 1 & 0 \\
C_{3} & 0 & C_{4} & D_{3} & 0 & D_{4}
\end{array}\right]
$$

where in case of $g_{12}$ the 4 by 4 matrix $g_{2}$ was denoted as $\left[\begin{array}{ll}A & B \\ C & D\end{array}\right]$, while in case of $g_{13}$ the 4 by 4 matrix $g_{2}$ was also denoted as $\left[\begin{array}{ll}A & B \\ C & D\end{array}\right]$, but with further specification of its 2 by 2 blocks: $A=$ $\left[\begin{array}{cc}A_{1} & A_{2} \\ A_{3} & A_{4}\end{array}\right], B=\left[\begin{array}{ll}B_{1} & B_{2} \\ B_{3} & B_{4}\end{array}\right], C=\left[\begin{array}{ll}C_{1} & C_{2} \\ C_{3} & C_{4}\end{array}\right], D=\left[\begin{array}{ll}D_{1} & D_{2} \\ D_{3} & D_{4}\end{array}\right]$. 


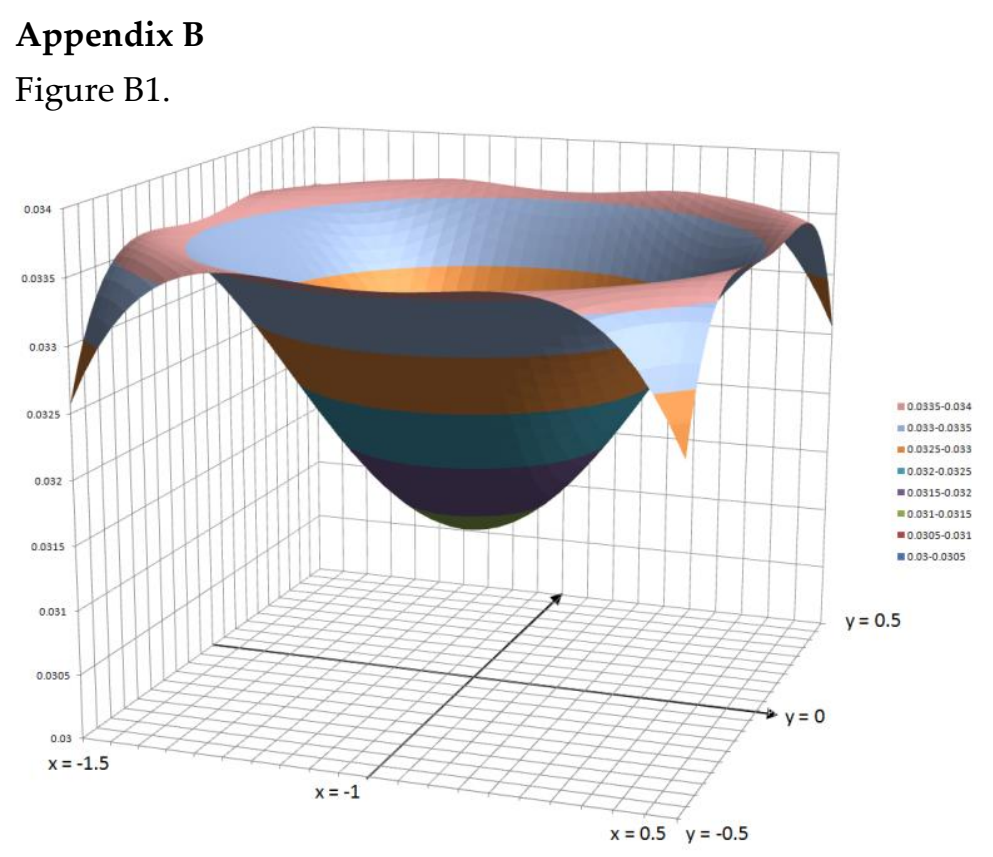

Figure B2.

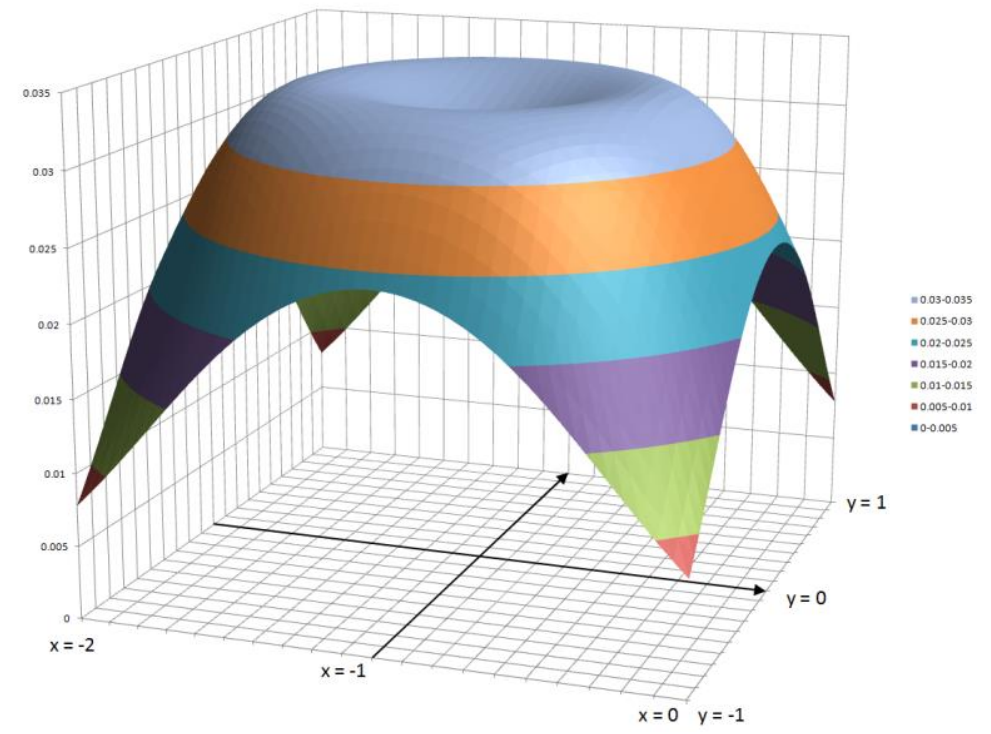

Figure B3.

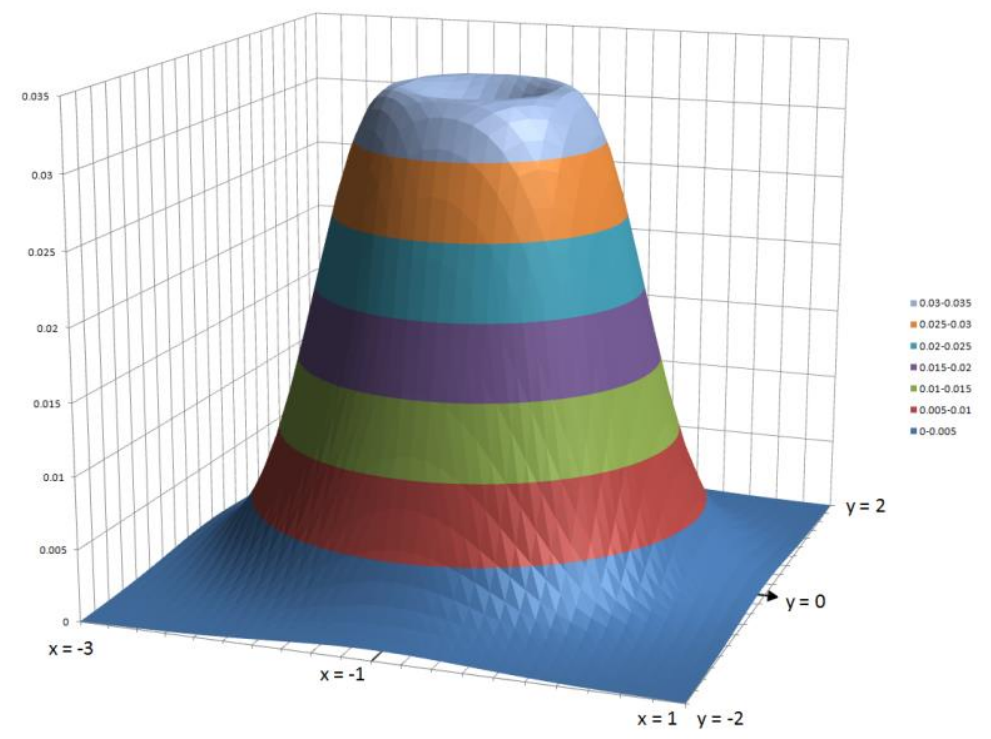


We thank Yu. Klevtsova for her help with analytic calculations which the Figures B1, B2, and B3 are based on.

\section{References}

1. Levichev, A.V. Towards a matrix multi-level model of quark-gluon media. J. Progressive Res. Math. 2016, 10, pp. 1493 --1496.

2. Levichev, A.V. One possible application of the Chronometric Theory of I.E. Segal: A toy model of quarks and gluons. J. Phys.: Conf. Ser. 2019, 1194012071

3. Segal, I.E. Is the Cygnet the quintessential baryon? Proc. Natl. Acad. Sci. 1991, 88, pp. $994-998$.

4. Wigner, E.P. On unitary representations of the inhomogeneous Lorentz group. Ann. of Math. (2) 1939, 40, pp. $149-204$.

5. Levichev, A.V.; Palyanov, A.Yu. On colors and electric charges of quarks: modeling in terms of groups U(n) and SU(n,n). Mathematical Structures and Modeling. 2020, 4(56), pp. 31--40, in Russian.

6. Levichev, A.V. On key properties of the intertwining operators ornament in the matrix multi-level model of the quark-gluon media. In Proceedings of the All-Russia conference with the international participation "Knowledge-Ontology-Theories" (KONT-2017), Novosibirsk, Sobolev Institute of Mathematics of the Siberian Division RAS, Russia, 6-8 October 2017; Volume 2, pp. 41-47.

7. Kon, M.; Levichev, A. Parallelization analysis of space-time bundles and applications in particle physics. In Proceedings of the All-Russia conference with the international participation "Knowledge-Ontology-Theories" (KONT-2019), Novosibirsk, Sobolev Institute of Mathematics of the Siberian Division RAS, Russia, 7--11 October 2019; pp. 385--392.

8. Levichev, A.; Palyanov, A. Standard charges of quarks determination in terms of the multi-level model. In Proceedings of the All-Russia conference with the international participation "Knowledge-Ontology-Theories" (KONT-2019), Novosibirsk, Sobolev Institute of Mathematics of the Siberian Division RAS, Russia, 7--11 October 2019; pp. 222--226.

9. Jakobsen, H.P.; Levichev, A.V.; Palyanov, A.Yu. The Wigner-Segal method as applied to the problem of quarks' and leptons' generations. . In Proceedings of the All-Russia conference with the international participation "Knowledge-Ontology-Theories" (KONT-2021), Novosibirsk, Sobolev Institute of Mathematics of the Siberian Division RAS, Russia, 8--12 November 2021; pp. 344--352. Online at http://math.nsc.ru/conference/zont/21/index.htm

10. Jakobsen, H.P.; Levichev, A.V. The representation of $\mathrm{SU}(2,2)$ which is interpreted as describing chronometric fermions (proton, neutrino, and electron) in terms of a single composition series. Rep. on Math. Phys. 2022, submitted.

11. Levichev, A.V. Segal's Chronometry: its development, application to the physics of particles and their interactions, further perspectives. Poisk matematicheskih zakonomernostei Mirozdania, M.M.Lavrent'ev, V.N.Samoilov, Eds.; GEO: Novosibirsk, Russia, 2010; Volume 7, pp. 66--99, in Russian.

12. Levichev, A.V. Pseudo-Hermitian realization of the Minkowski world through DLF theory. Physica Scripta. 2011, 83, pp. 1-9. Online at stacks.iop.org/PhysScr/83/015101

13. Paneitz, S.M.; Segal, I.E.; Vogan, D.A., Jr. Analysis in space-time bundles, IV. J. Funct. Anal. 1987, 75, pp. 1--57.

14. Jakobsen, H.P. Intertwining differential operators for Mp(n;R) and SU(n; n). Trans. Amer. Math. Soc. 1978,246, pp. $311--337$.

15. Breidenbach, M. et al. Observed Behavior of Highly Inelastic Electron-Proton Scattering. Phys. Rev. Lett. 1969, 23, pp. 935--939.

16. Jakobsen, H.P.; Vergne, M. Wave and Dirac Operators and Representations of the Conformal Group. J. Funct. Anal. 1977, 24, pp. 52-106.

17. Barut, A.O. A. Return to 1932: proton, electron and neutrino as true elementary constituents of leptons, hadrons and nuclei. Quantum Theory and the Structures of Time and Space, 1981, Volume 4, pp. 152--163. Carl Hanser Press, Munich.

18. Varadarajan, V.S. Geometry of Quantum Theory; Springer: New York, USA, 1985.

19. Faessler, M.A. Weinberg Angle and Integer Electric Charges of Quarks. https://arxiv.org/abs/1308.5900 , 2013.

20. Paneitz, S.M.; Segal, I.E. Analysis in space-time bundles I: General considerations and the scalar bundle. J. Funct. Anal. 1982, 47, pp. 78-142.

21. Voloshin, M.B.; Ter-Martirosyan, K.A. Theory of gauge interactions of elementary particles; EnergoAtomIzdat: Moscow, 1984, in Russian.

22. Malaev, M.V. Birth of $\varphi$-mesons in $p-p, p$ - $\mathrm{Pb}$, and Pb-Pb collisions in the ALICE experiment at LHC. PhD, Konstantinov Institute of nuclear Physics, Saint Petersburg, Russia, 2015, in Russian.

23. Weber, F.; Farrell, D; et al. Phases of Hadron-Quark Matter in (Proto) Neutron Stars. 2019. arXiv:1907.06591v1 [nucl-th]

24. Levichev, A.V.; Klevtsova, Yu.Yu.; Palyanov, A.Yu. Alexandrov-110 and a contribution to Chronometry. MSEM. 2022, submitted, in Russian.

25. Burkert, V.D.; Elouadrhiri, L.; Girod, F.X. The pressure distribution inside the proton. Nature. 2018, 557, pp. 396-399 https://doi.org/10.1038/s41586-018-0060-z

26. Khabarova, K.Yu.; Kolachevsky, N.N. Proton charge radius. 2021. Uspekhi Fizicheskikh Nauk. 191 (10) pp. 1095-1106, in Russuan, https://ufn.ru/ru/articles/2021/10/d/

27. Nekrasov, M.L. On the Shape of Nucleons at High Energies. Particles. 2021, 4(3), pp. 381-390.

28. Segal, I. E. Covariant chronogeometry and extreme distances. III. Macro-micro relations. Int. J. Theor. Phys. 1982, 21, pp. 851869. 